\title{
Synergistic effect of HMGB1 knockdown and cordycepin in the K562 human chronic myeloid leukemia cell line
}

\author{
XI CHEN*, YING WANG*, JUAN LIU, PING XU, XIAO-MIN ZHANG, YAO-YAO TIAN, \\ YAN-MING XUE, XIN-YU GAO, YAO LIU and JING-HUA WANG \\ Department of Hematology, The Second Affiliated Hospital, Harbin Medical University, \\ Harbin, Heilongjiang 150086, P.R. China
}

Received August 26, 2014; Accepted May 13, 2015

DOI: $10.3892 / \mathrm{mmr} .2015 .3928$

\begin{abstract}
The high-mobility group box 1 (HMGB1) protein is a DNA-binding nuclear protein, which is overexpressed in leukemia cells. Cordycepin is characterized by strong antileukemic properties and is regarded as an effective natural compound for leukemia therapy. The aim of the present study was to investigate the impact of HMGB1 knockdown and cordycepin treatment on proliferation, apoptosis, reactive oxygen species (ROS) levels and adhesion of K562 human chronic myeloid leukemia cells. The Cell Counting kit- 8 assay was used to determine the proliferation of K562 cells. The cell cycle and apoptosis of K562 cells was determined using flow cytometric analysis. In addition, a cell adhesion assay was performed. Western blotting was used to determine the protein expression of cyclooxygenase 2, Bax, receptor for advanced glycation end-products and Bcl-2. The data collected demonstrated that HMGB1 knockdown combined with cordycepin treatment had significant anti-proliferative and pro-apoptotic effects. In addition, it increased the ROS levels and reduced the adhesion of K562 cells. It was also identified that HMGB1 knockdown had synergistic effects with cordycepin, which aided in accelerating apoptosis, and inhibiting proliferation and adhesion in chronic myeloid leukemia cells. These results indicated that HMGB1 may be used as a potential therapeutic target, with cordycepin having potential as an auxiliary drug. Therefore, it is suggested that HMGB1 knockdown and corycepin treatement may present a promising therapeutic strategy for leukemia.
\end{abstract}

Correspondence to: Mrs. Jing-Hua Wang, Department of Hematology, The Second Affiliated Hospital, Harbin Medical University, 246 Xue-Fu Road, Harbin, Heilongjiang 150086, P.R. China

E-mail: jinghuawang123@163.com

${ }^{*}$ Contributed equally

Key words: high-mobility group box 1, cordycepin, chronic myeloid leukemia, k562, proliferation, apoptosis

\section{Introduction}

Chronic myeloid leukemia (CML) is a hematopoietic stem cell disorder; which has serious implications on health and life expectancy (1). It is diagnosed by the presence of a specific abnormality karyotype Philadelphia $(\mathrm{Ph})$ chromosome, harboring the BCR-ABL oncogene (2). CML follows progression from a chronic phase to an accelerated phase or to a rapidly fatal blast crisis within 3-5-years in patients (3). It has been reported that imatinib, nilotinib and dasatinib can be used in the treatment of patients with CML (4). At present, treatment strategies for leukemia remain unsatisfactory, thus it is imperative to fully elucidate the molecular mechanisms of leukemia. It will be undoubtedly a general trend that molecular therapy combined with anti-leukemia natural compound are employed in leukemia treatment (5).

High mobility group box 1 (HMGB1), a DNA-binding nuclear protein predominantly involved in the inflammatory response, has a close association with the growth of tumor cells, invasion and metastasis (6,7). It is the prototypic damage-associated molecular pattern (DAMP) molecule and has been implicated in several inflammatory disorders (8). High levels of HMGB1 have been previously detected in leukemia cells (9). HMGB1 is known to be a type of anti-apoptotic binding protein and its over-expression is able to inhibit cell apoptosis, leading to the occurrence of leukemic growth (10). The upregulation of HMGB1 mRNA expression has been identified in a number of tumor types (11-13). In addition, HMGB1 has been reported to reduce the sensitivity of K562 human myeloid leukemia cells to anticancer drugs (14). Therefore, targeting the HMGB1 ligand or its receptor represents an important potential application in leukemia therapy. Cordycepin (3'-deoxyadenosine) is a key bioactive component isolated from $C$. militaris. It has been reported to possess numerous pharmacological activities, including immunological stimulation, anticancer and antileukemic effects (15-17). Previous studies have indicated that cordycepin has a range of molecular targets and influences numerous biochemical and molecular processes (18-20). However, the molecular mechanisms of cordycepin on leukemia remain to be fully elucidated.

In the present study, HMGB1 knockdown cells were established using the lentiviral infection method. The effects of HMGB1 knockdown in combination with cordycepin treat- 
ment on proliferation, apoptosis, reactive oxygen species (ROS) and adhesion of the K562 leukemia cell line were evaluated. In addition, whether cordycepin exhibited synergistic action with the HMGB1 knockout was investigated, which aimed to provide insight into CML treatment.

\section{Materials and methods}

Cell culture, transfection and treatment. The K562 and 293T human CML cell lines were purchased from the American Type Culture Collection (Manassas, VA, USA) and cultured in Dulbecco's modified Eagle's medium (DMEM; Invitrogen Life Technologies, Carlsbad, CA, USA) containing 10\% (v/v) heat-inactivated fetal bovine serum (FBS; Gibco Life Technologies- Carlsbad, CA, USA), 100 mg/ml streptomycin and $100 \mathrm{U} / \mathrm{ml}$ penicillin (Beyotime Institute of Biotechnology, Haimen, China) at $37^{\circ} \mathrm{C}$ in a humidified $5 \% \mathrm{CO}_{2}$ atmosphere. Cells with HMGB1 high-expression were screened for transfection by means of western blotting. The small interfering RNA (siRNA)-HMGB1 plasmid was provided by JRDUN Biotechnogy (Shanghai) Co., Ltd. (Shanghai, China). The HMGB1 plasmid (3 mg) or mock-vehicle were transfected into $\mathrm{K} 562$ cell lines in 6 -well plates $(1 \mathrm{mg} / \mathrm{ml})$ using the pCMVG-NR-U6-shRNA lentiviral vector (Shanghai Genechem Co., Ltd., Shanghai, China), according to the manufacturer's instructions. Cells were treated with cordycepin $(30 \mu \mathrm{mol} / \mathrm{l})$ for $48 \mathrm{~h}$, and then were subjected to cell assays.

Cell viability assay. For the cell viability assay, cells $\left(5 \times 10^{5} / \mathrm{ml}\right)$ were seeded into 96-well plates and cultured for 0, 24, 48 and $72 \mathrm{~h}$ at $37^{\circ} \mathrm{C}$. Subsequently, $100 \mu \mathrm{l}$ serum free DMEM containing 10\% Cell Counting kit-8 (CCK-8; Beyotime Institute of Biotechnology) reagent (v/v) was added into each well and the cells were cultured for $1 \mathrm{~h}$ in $5 \% \mathrm{CO}_{2}$ at $37^{\circ} \mathrm{C}$. Finally, optical density values (OD) were read at $450 \mathrm{~nm}$ using a SpectraMax ${ }^{\circledR}$ i3x microplate reader (Molecular Devices, Sunnyvale, CA, USA).

Detection of cell cycle and apoptosis by flow cytometric analysis. The cell cycle was assessed according to the percentage of cells with DNA using the propidium iodide (PI) staining technique. With or without cordycepin treatment for $48 \mathrm{~h}$, cells $\left(5 \sim 10 \times 10^{4}\right)$ were harvested and stained using an Annexin V-fluorescein isothiocyanate/PI kit (BD Biosciences, Franklin Lakes, NJ, USA). Staining was performed according to the manufacturer's instructions. The apoptosis of K562 cells was determined by flow cytometric analysis using a FACSCaliber flow cytometer (BD Biosciences).

Detection of ROS. The generation of ROS was assessed by flow cytometry. In brief, cells $\left(5 \times 10^{4}\right.$ cells/well) were cultured and washed with phosphate-buffered saline (PBS) and resuspended in complete medium followed by incubation with $50 \mu \mathrm{M}$ dihydrorhodamine (DHE; Vigorous Biotechnology, Beijing, China) for $30 \mathrm{~min}$ at $37^{\circ} \mathrm{C}$. ROS fluorescence intensity was determined by flow cytometry with excitation at $490 \mathrm{~nm}$ and emission at $520 \mathrm{~nm}$.

Cell adhesion assay. The cell adhesion assay was conducted in 12 -well plates according to the method described previ- ously (21). The wells were precoated with fibronectin (Sigma-Aldrich, St. Louis, MO, USA) overnight at room temperature. K562 cells were harvested and re-suspended in DMEM containing $10 \% \mathrm{FBS}$; then, cells were added ( $2 \times 10^{5} /$ well) to each well and incubated at $37^{\circ} \mathrm{C}$ for $1 \mathrm{~h}$. The wells were washed twice with warm PBS to remove the unattached cells, and the attached cells were fixed with methanol for $15 \mathrm{~min}$ and stained with crystal violet (Sigma-Aldrich) for $20 \mathrm{~min}$. Subsequent to staining, the cells were observed using an optical BX51 microscope (Olympus, Tokyo, Japan).

Western blot analysis. Following the above treatments, cells were harvested and lysed in radioimmunoprecipitation assay buffer (Beyotime Institute of Biotechnology) and protease inhibitor cocktail (Sigma-Aldrich) for $10 \mathrm{~min}$ at $4^{\circ} \mathrm{C}$. The protein concentration was determined using Bicinchoninic Acid Protein Assay Reagent (Thermo Fisher Scientific, Waltham, MA, USA). Subsequently, equal quantities of denatured protein $(20 \mu \mathrm{g})$ were separated on 10\% SDS-PAGE gels (Beyotime Institute of Biotechnology), then transferred to nitrocellulose membranes (Pall Corporation, Pensacola, FL, USA) and incubated overnight at $4^{\circ} \mathrm{C}$ with a cyclooxygenase 2 (COX-2, Abcam, Cambridge, MA, USA, cat. no. Ab62331, 1:500), receptor for advanced glycation end products (RAGE, Abcam, cat. no. Ab54741, 1:1,000), Bax (Santa Cruz Biotechnology, Inc., Dallas, TX, USA, cat. no. Sc-493, 1:150) and Bcl-2 (Santa Cruz Biotechnology, Inc., cat. no. Sc-492, 1:100) monoclonal primary antibodies, followed by incubation with a goat anti-mouse/horseradish peroxidase conjugated secondary antibody and chemiluminescence detection (ECL; Millipore, Billerica, MA, USA). To ensure equivalent protein loading, antibodies targeted against GAPDH were used, and the protein expression levels were normalized to GAPDH. The western blots presented in the figures are representative of three independent experiments unless otherwise indicated.

Statistical analysis. All data are presented as the mean \pm standard deviation of three determinations. For statistical analysis, Prism, version 5.0 (GraphPad Software, Inc., La Jolla, CA, USA) was used. $\mathrm{P}<0.05$ was considered to indicate a statistically significant difference.

\section{Results}

HMGB1 knockdown and cordycepin inhibit the proliferation of K562 cells. In order to evaluate the effects of HMGB1 knockdown with/without cordycepin treatment on the proliferation of K562 cells, the OD $(450 \mathrm{~nm})$ value of K562 cells was detected using the CCK-8 assay. As presented in Table I, the proliferation of K562 cells in the mock with cordycepin, siRNA-HMGB1 and siRNA-HMGB1 with cordycepin groups was significantly inhibited at 24,48 and $72 \mathrm{~h}(\mathrm{P}<0.01)$, when compared with the mock group. The results of the present study indicate that the knockdown of HMGB1 may significantly inhibit the proliferation of K562 cells.

Cell cycle analysis demonstrated that the mock cells treated with cordycepin, HMGB1 knockdown cells and HMGB1 knockdown cells treated with cordycepin exhibited $\mathrm{G}_{1}$ arrest prior to the appearance of apoptosis in K562 cells. The cells in the $S$ and $G_{2}$ phases were additionally reduced compared with the mock (Fig. 1A and B). 
Table I. Effect of HMGB1 knockdown on proliferation of the K562 cell line.

\begin{tabular}{lcccr}
\hline Group & $0 \mathrm{~h}$ & $24 \mathrm{~h}$ & $48 \mathrm{~h}$ & $72 \mathrm{~h}$ \\
\hline Mock & $0.317 \pm 0.0025$ & $0.531 \pm 0.0062$ & $0.839 \pm 0.0070$ & $1.054 \pm 0.0269$ \\
Mock + cordycepin & $0.317 \pm 0.0055$ & $0.476 \pm 0.0045^{\mathrm{a}}$ & $0.671 \pm 0.0060^{\mathrm{a}}$ & $0.838 \pm 0.0096^{\mathrm{a}}$ \\
siRNA-HMGB1 & $0.316 \pm 0.0055$ & $0.460 \pm 0.0032^{\mathrm{a}}$ & $0.638 \pm 0.0096^{\mathrm{a}}$ & $0.771 \pm 0.0060^{\mathrm{a}}$ \\
siRNA-HMGB1 + cordycepin & $0.315 \pm 0.0059$ & $0.417 \pm 0.0053^{\mathrm{a}}$ & $0.561 \pm 0.0064^{\mathrm{a}}$ & $0.661 \pm 0.0064^{\mathrm{a}}$
\end{tabular}

siRNA, small interfering RNA; HMGB1, high-mobility group box 1 protein.

A Mock Mock+cordycepin
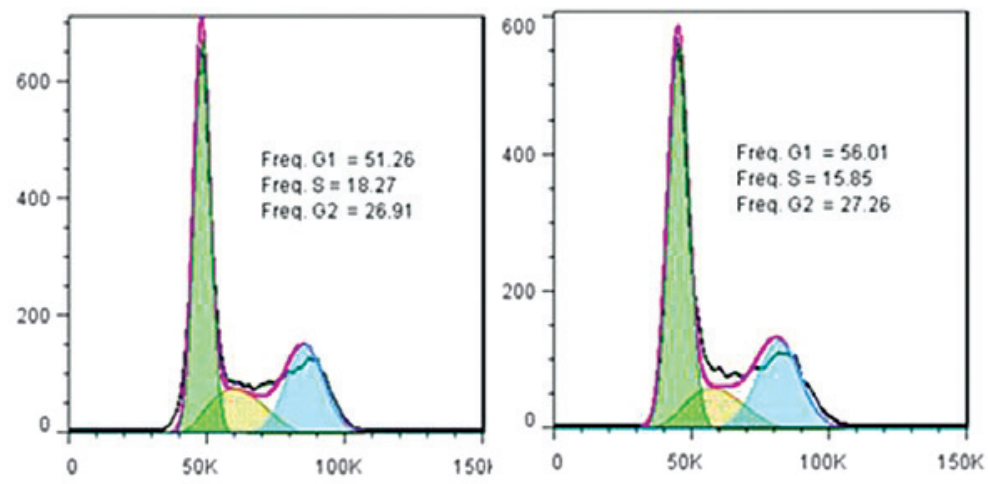

siRNA-HMGB1

siRNA-HMGB1+cordycepin
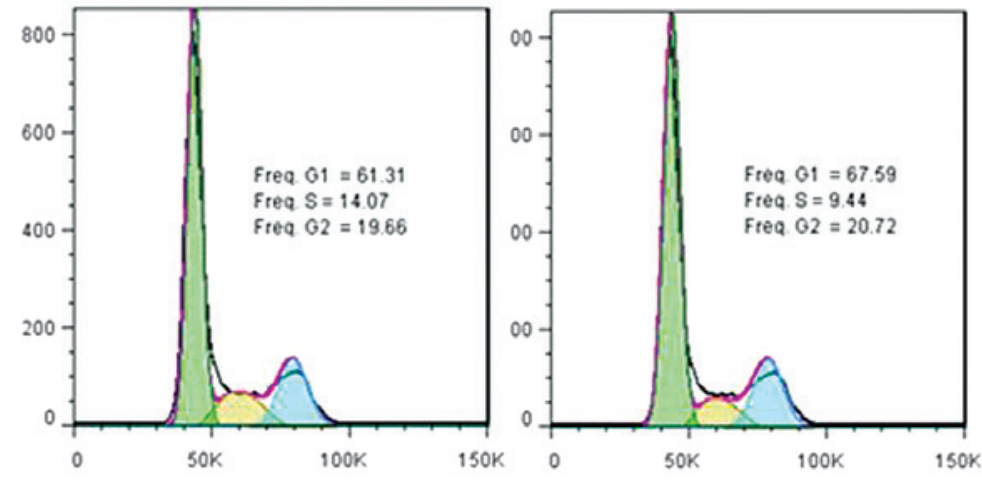

B

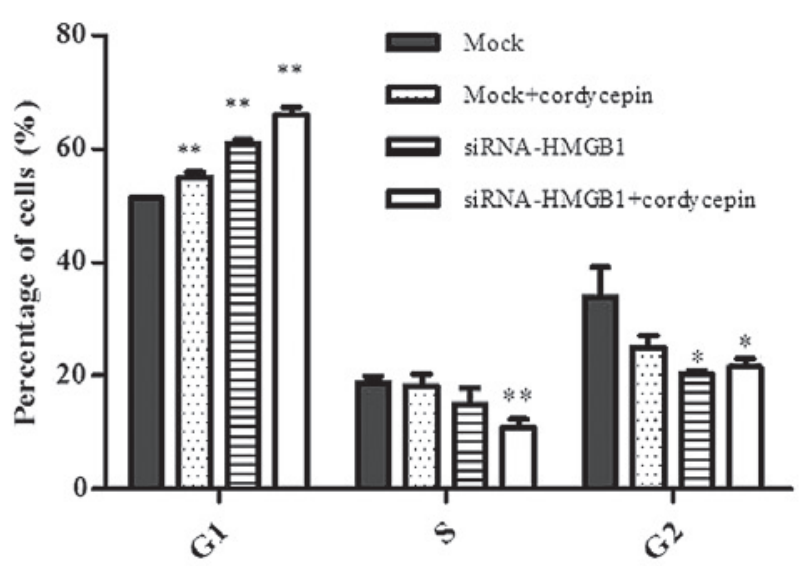

Figure 1. Results of cell cycle analysis in the mock (cells treated with the control vector), mock + cordycepin, siRNA-HMGB1 (HMGB1 gene knockdown) and siRNA-HMGB1 + with cordycepin groups. (A) Representative results for cell cycle distribution. (B) Data pooled from three independent experiments illustrating the cell-cycle distribution. ${ }^{* *} \mathrm{P}<0.01$, vs. mock group; ${ }^{*} \mathrm{P}<0.05$, vs. mock group. siRNA, small interfering RNA; HMGB1, high-mobility group box 1 protein. 
A

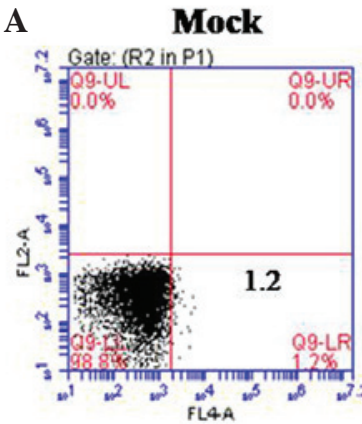

B
Mock+cordycepin
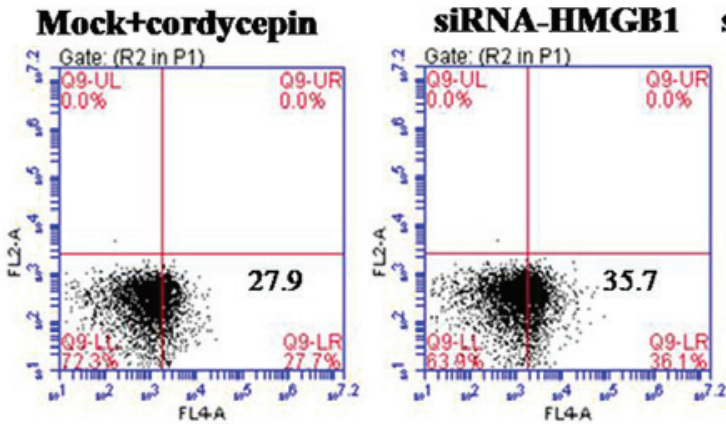

siRNA-HMGB1+cordycepin
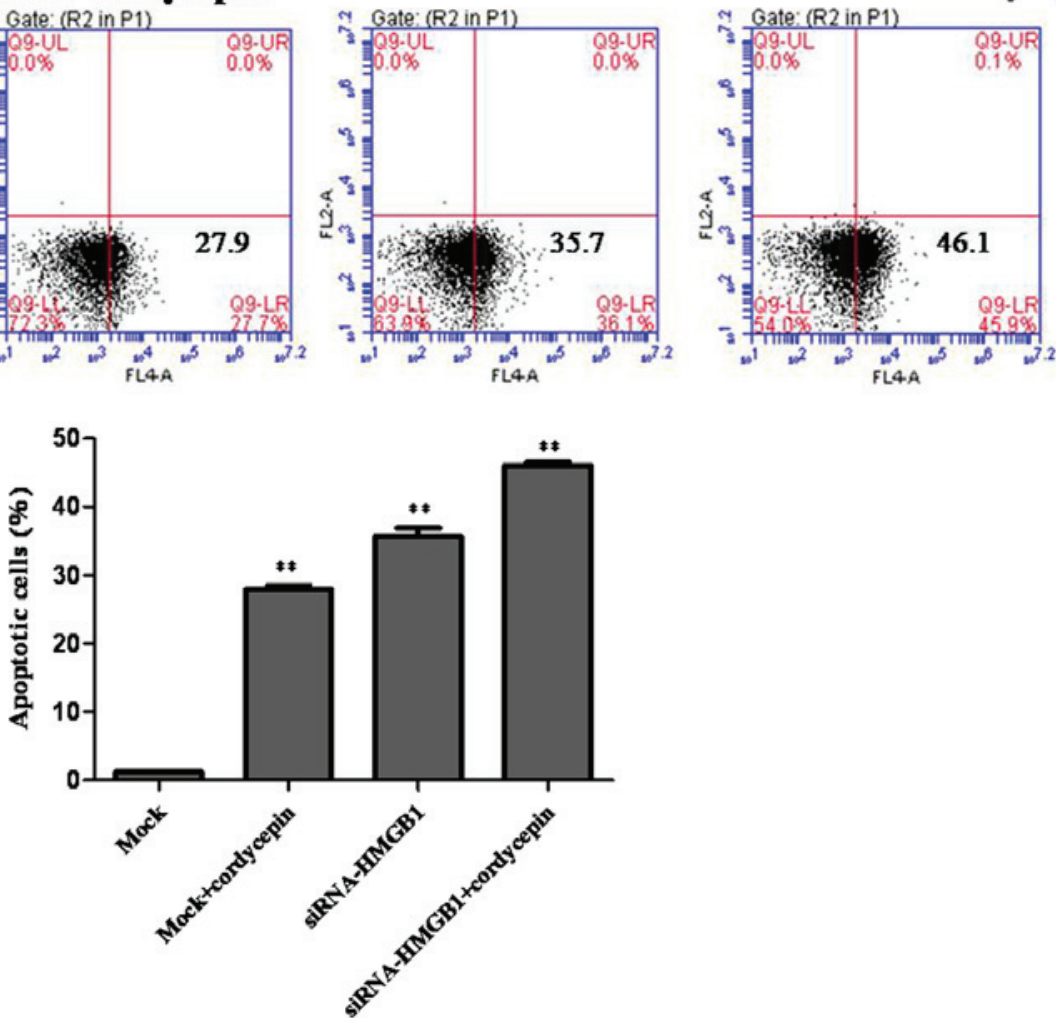

Figure 2. Results of the apoptosis assay from flow cytometric analysis. (A) Representative flow cytometry results. (B) Apoptosis assay results. The data are presented as the mean \pm standard deviation, $\mathrm{n}=3,{ }^{* *} \mathrm{P}<0.01$, vs. mock group. The percentage of apoptotic cells identified were $1.2 \%$ in the mock group, $27.9 \%$ in the mock + cordycepin group, $35.7 \%$ in the siRNA-HMGB1 group and $46.1 \%$ in the siRNA-HMGB1 + cordycepin group. Mock, cells treated with the control vector; siRNA-HMGB1, HMGB1 knockdown in K562 cells. siRNA, small interfering RNA; HMGB1, high-mobility group box 1 protein.
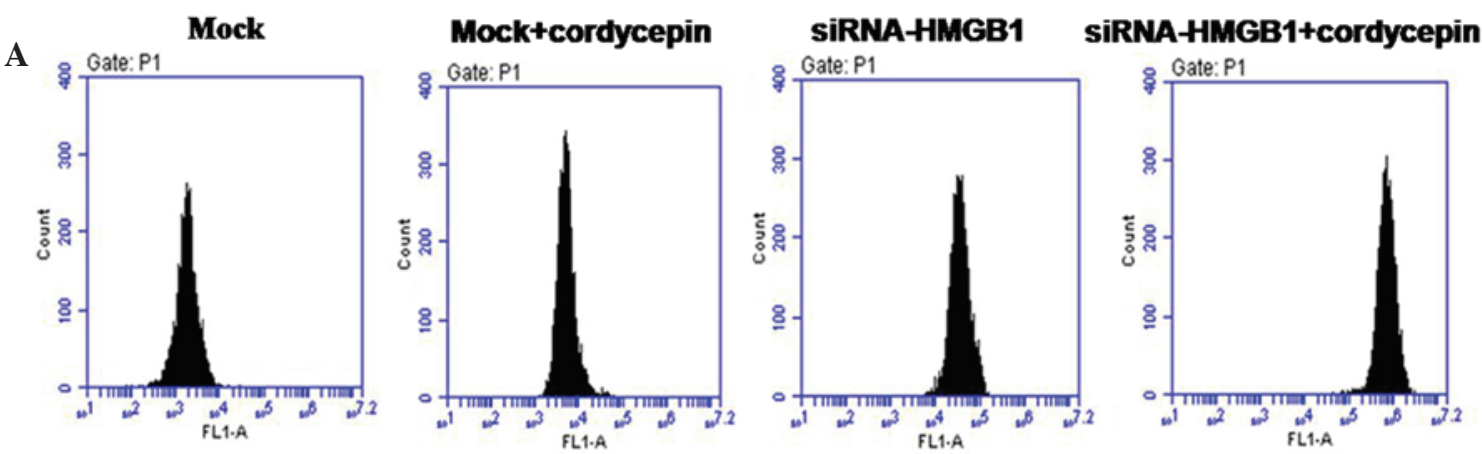

B

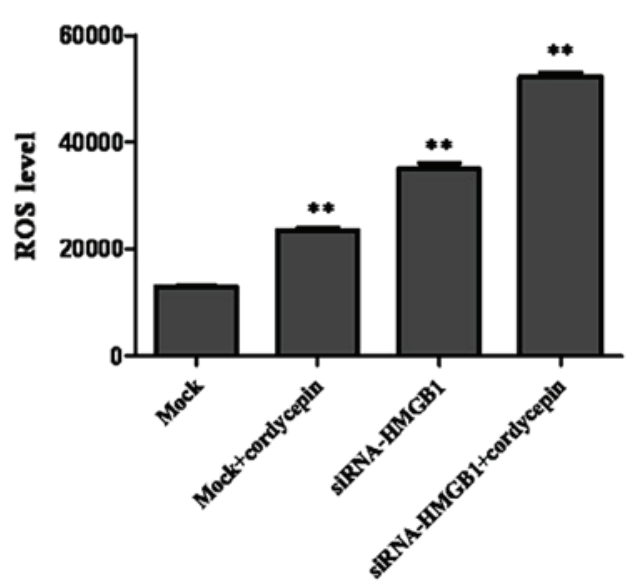

Figure 3. Level of ROS generation in K562 cells. (A) HMGB1 knockdown was treated with cordycepin and ROS generation was examined by flow cytometry. (B) Treatments compared with mock. ROS levels were observed to significantly increase. ${ }^{* *} \mathrm{P}<0.01$ vs. mock. ROS, reactive oxygen species; siRNA, small interfering RNA; HMGB1, high-mobility group box 1 protein. 
A

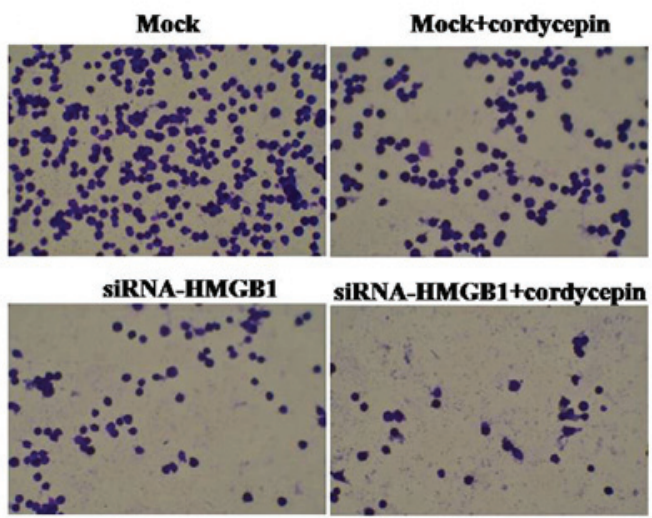

C

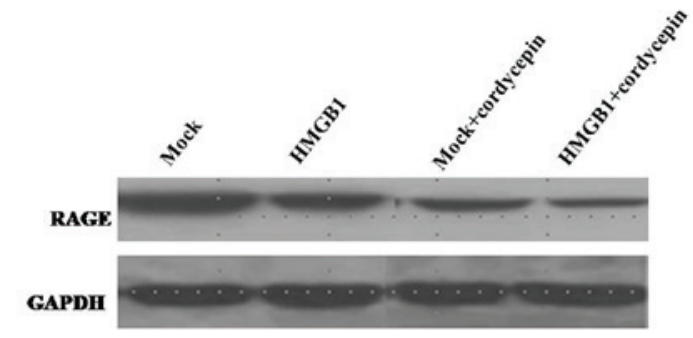

B
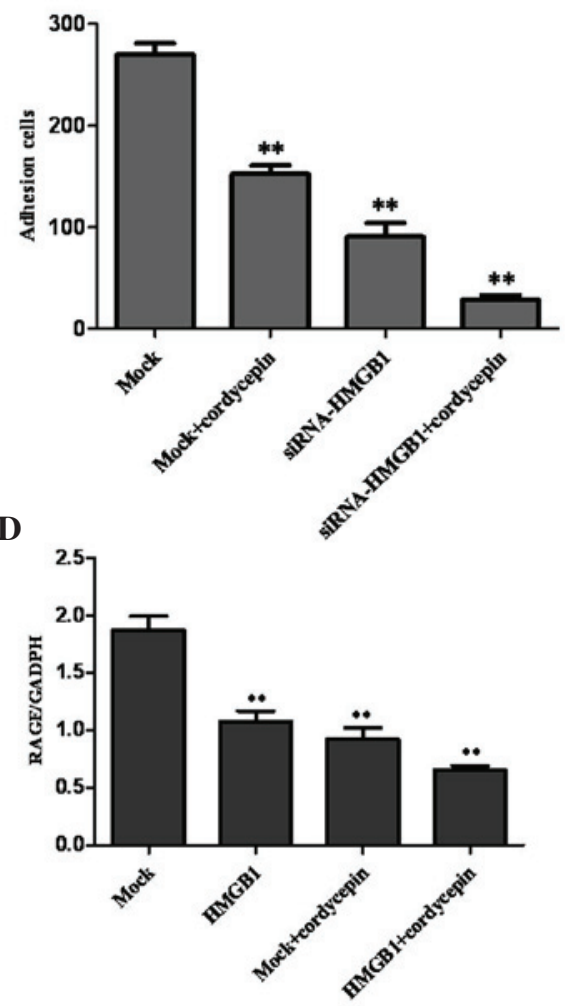

Figure 4. Results of cell adhesion assay with cordycepin treatment. (A) Adherent cells were stained with crystal violet for 20 min and images were captured under light microscopy (magnification, x200). (B) Three random fields of adherent cells were counted and summarized. Data are presented as the mean \pm standard deviation, $n=3,{ }^{* *} \mathrm{P}<0.01$ vs. mock group. (C) The expression levels of RAGE. (D) The expression level of RAGE was normalized to GAPDH and analyzed by grayscale. The HMGB1 knockdown treated with cordycepin has extremely decreased compared with the mock group, ${ }^{* *} \mathrm{P}<0.01$. Mock, cells treated with the control vector; siRNA-HMGB1, HMGB1 knockdown in K562 cells. RAGE, receptor for advanced glycation end products. siRNA, small interfering RNA; HMGB1, high-mobility group box 1 protein.

HMGB1 knockdown and cordycepin encourage the apoptosis of $K 562$ cells. To estimate the anti-proliferative effect of HMGB1 knockdown, cordycepin treatment and the combined action of HMGB1 knockdown + cordycepin in contributing to the inhibition of K562 cell apoptosis, flow cytometric analysis was performed. From the results obtained, the mock cells treated with cordycepin, HMGB1 knockdown cells and HMGB1 knockdown cells treated with cordycepin exhibited significantly increased apoptosis rates of K562 cells compared with the mock (Fig. 2).

ROS generation was identified following HMGBI and cordycepin treatment. Excessive generation of ROS in the cell is also shown to induce apoptosis (22). The fluorescent probe DHE was used to determine the levels of ROS production in K562 cells. As presented in Fig. 3, rapid generation of ROS was detected following HMGB1 knockdown and cordycepin treatment.

$H M G B 1$ knockdown and cordycepin inhibit the adhesion of $B G C-823$ cells by downregulating RAGE levels. As presented in Fig. 4A and B, cell adhesion was significantly inhibited, compared with that in the mock group. The present study indicated that the knockdown of HMGB1 was able to significantly suppress adhesion of K562 cells. RAGE is a receptor for DAMP molecules and reacts in particular with HMGB1 (23). RAGE acts as an adhesion molecule and the expression level was observed to be significantly downregulated; thus, suggesting that HMGB1 knockdown and treatment with cordycepin are able to inhibit adhesion by downregulating RAGE expression (Fig. 4C and D).

HMGB1 knockdown and cordycepin inhibit proliferation of K562 cells by downregulating COX-2 levels. COX-2 is a key enzyme in arachidonic acid metabolism, which serves an essential role in cell proliferation and apoptosis (24). As presented in Fig. 5, the levels of COX-2 expression in normal cells were higher in cordycepin-treated cells, HMGB1 knockdown cells and cordycepin-treated knockdown cells. The result may indicate that HMGB1 and cordycepin inhibit proliferation of K562 cells by downregulating COX-2 expression.

HMGB1 knockdown and cordycepin promote K562 cell apoptosis by regulating apoptotic factors. In order to elucidate the mechanism of K562 cell apoptosis induced by HMGB1, the expression levels of the apoptosis-associated proteins, Bax and $\mathrm{Bcl}-2$, were detected by western blot analysis. Bax was identified to exhibit a positive role on apoptosis, while $\mathrm{Bcl}-2$ is an anti-apoptotic protein. Bax was upregulated in cordycepin-treated cells, siRNA-HMGB1 plasmid-transfected cells and transfected cells seeded with cordycepin, compared with mock cells. Conversely, Bcl-2 expression was observed to be lower than that in the mock cells. The relative value 


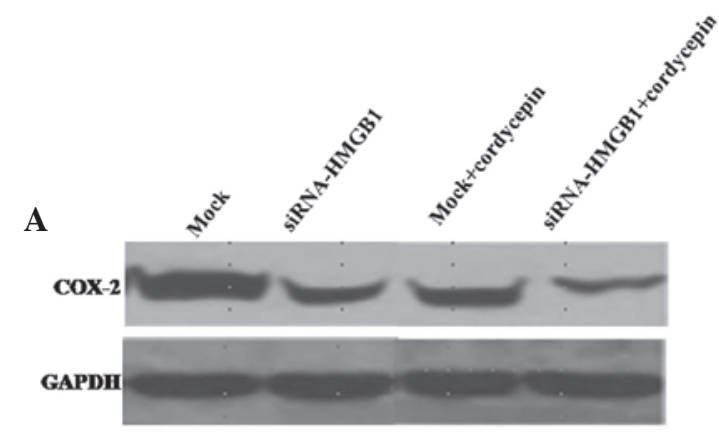

B

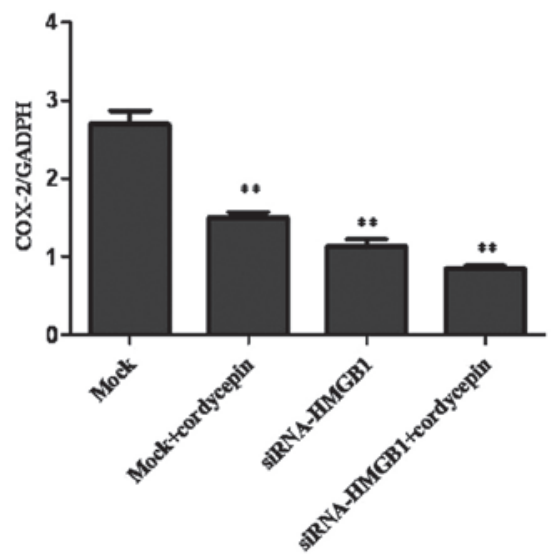

Figure 5. COX-2 expression levels. GAPDH was detected as a loading control. (A) Western blot analysis of COX-2 expression. (B) Quantification of COX-2 expression levels. The data are presented as the mean \pm standard deviation, $\mathrm{n}=3,{ }^{* *} \mathrm{P}<0.001$ vs. mock. COX-2, cyclooxygenase 2 ; siRNA, small interfering RNA; HMGB1, high-mobility group B1 protein.

of Bax/Bcl-2 is presented in Fig. 6. The Bax/Bcl-2 value of knockdown cells seeded with cordycepin was notably higher than that in the mock group. These results indicated that HMGB1 knockdown and cordycepin treatment promoted K562 cell apoptosis by regulating apoptotic factors.

\section{Discussion}

Leukemia is a malignant hematopoietic disorder characterized by the clonal proliferation of hematopoietic stem cells and immature myeloid precursors. Accompanied with complex symptoms, difficulty in treatment and a poor prognosis, leukemia is a great threat to a patients' health and survival chances. Treatment of leukemia predominantly consists of chemotherapy, radiotherapy, bone marrow transplantation or stem cell transplantation and targeted therapy (25). Molecular therapy and treatment using natural products for $\mathrm{CML}$ are currently areas of research focus. HMGB1, a protein expressed abundantly in CML cells, has been reported to serve a critical role in CML development and progression (14), while cordycepin has been demonstrated to exhibit antileukemia properties (26). The current study investigated the effects on proliferation, apoptosis, ROS levels and adhesion following HMGB1 knockdown, cordycepin treatment and a combination of the two in order to provide a theoretical basis for early clinical diagnosis and intervention.

In the current study, the combination of HMGB1 knockdown and cordycepin treatment was observed to effectively
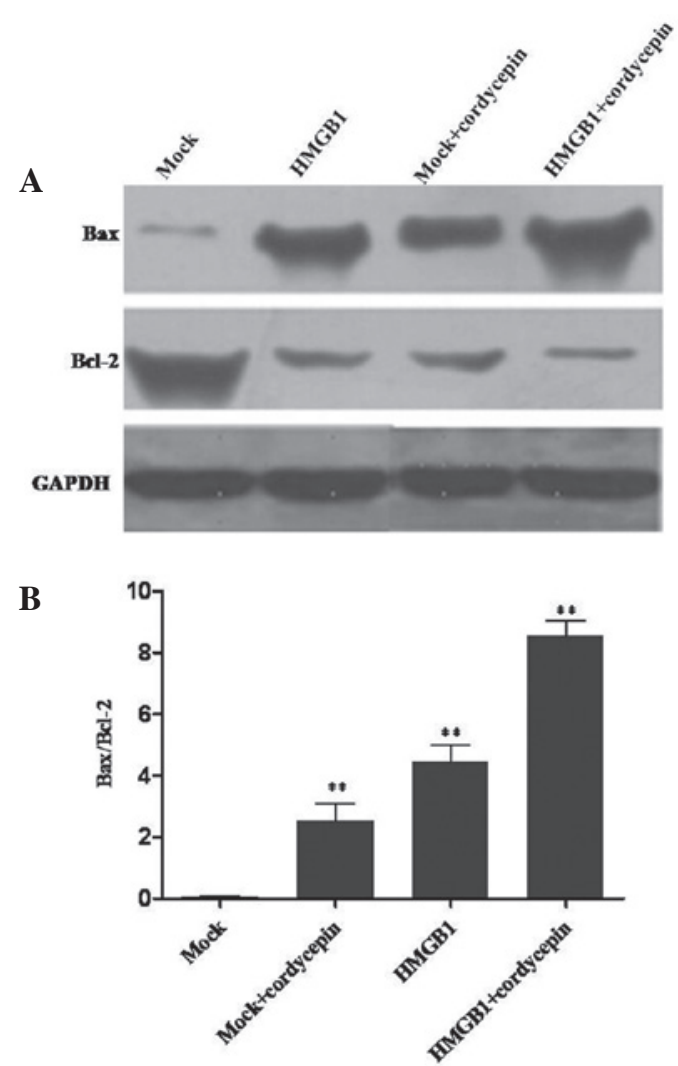

Figure 6. Expression levels of apoptosis-associated genes. GAPDH was detected as a loading control. (A) Western blot analysis of Bax and Bcl-2 expression. (B) Quantification of Bax and Bcl-2 expression levels. The data are presented as the mean \pm standard deviation, $n=3,{ }^{* *} \mathrm{P}<0.01$ vs. mock. siRNA, small interfering RNA; HMGB1, high-mobility group B1 protein.

suppress the proliferation of K562 human CML cells. The impact of HMGB1 knockdown with cordycepin on the cell cycle demonstrated that HMGB1, cordycepin and their combination arrested the cell cycle at $\mathrm{G}_{1}$ phase. Previous studies have indicated that COX-2 expression activates certain signaling pathways that control proliferation and apoptosis (27-29). The expression of COX-2 in the experimental group was downregulated compared with the mock group, which suggested that HMGB1 knockdown and cordycepin inhibited cell proliferation via the downregulation of COX-2 expression.

In addition, the present study indicated that knockdown of HMGB1 significantly inhibited the adhesion of K562 cells. The combination of HMGB1 knockdown and cordycepin was observed to exhibit improved action compared with either treatment alone. RAGE is a predominant receptor in mediating the effects of HMGB1 (30). It has been reported that RAGE evolved from a cell adhesion molecule family and acts as an adhesion molecule in mammalian cells (31). The HMGB1 knockdown and cordycepin-treated groups exhibited downregulated expression of RAGE compared with the mock group. The interaction of HMGB1 with RAGE can influence adhesion in HMGB1 knockdown and cordycepin-treated cells.

Apoptosis, the process of programmed cell death, is the common mechanism that chemotherapies, cytotoxic agents or radiation therapies target to induce cancer cell death. HMGB1 knockdown, cordycepin and their combination increase K562 cellular apoptosis. The combination of HMGB1 knockdown 
and cordycepin induced apoptosis according to the flow cytometric analysis, with a greater significance than either treatment alone. Bax and Bcl-2 are proteins that serve important roles in cell apoptosis $(32,33)$. Evaluation of the expression levels of apoptotic proteins Bax and Bcl-2 further confirmed the conclusion that the combination of HMGB1 knockdown and cordycepin synergistically induced cellar apoptosis. The levels of ROS were additionally observed to increase in the treatment groups. ROS are free radicals, for example $\mathrm{O}_{2-}, \mathrm{OH}$, $\mathrm{H}_{2} \mathrm{O}_{2}$ and ${ }^{1} \mathrm{O}_{2}$, which have numerous effects on signal transduction. ROS production and consequential oxidative stress have been previously implicated in cellular apoptosis (34). In the current study, the ROS levels were observed to be significantly increased in the HMGB1 knockdown and cordycepin groups, which may be the cause of the increased apoptosis. The above results indicated that HMGB1 knockdown promoted the apoptosis of K562 cells by regulating apoptotic factors and the ROS levels, and that combination therapy with cordycepin resulted in increased apoptosis.

In conclusion, the current study identified that HMGB1 knockdown in combination with cordycepin resulted in increase apoptosis, decreased proliferation, and reduced adhesion of human CML K562 cells, as well as increased ROS, and the corresponding mechanisms were investigated. These observations may provide insight towards fully elucidating the molecular mechanism of CML progression and pathogenesis, thus benefiting the development of therapeutic strategies for the disease.

\section{References}

1. Druker BJ: STI571 (Gleevec) as a paradigm for cancer therapy. Trends Mol Med 8 (Suppl 4): S14-S18, 2002.

2. Melo JV, Hughes TP and Apperley JF: Chronic myeloid leukemia. Hematology (Am Soc Hematol Educ Program) 2003: 132-152, 2003

3. Baran Y, Salas A, Senkal CE, Gunduz U, Bielawski J, Obeid LM and Ogretmen B: Alterations of ceramide/sphingosine 1-phosphate rheostat involved in the regulation of resistance to imatinib-induced apoptosis in K562 human chronic myeloid leukemia cells. J Biol Chem 282: 10922-10934, 2007.

4. Cagnetta A, Garuti A, Marani C, et al: Evaluating treatment response of chronic myeloid leukemia: Emerging science and technology. Curr Cancer Drug Targets 13: 779-790, 2013.

5. Ji J, Wang HS, Gao YY, Sang LM and Zhang L: Synergistic antitumor effect of KLF4 and curcumin in human gastric carcinoma cell line. Asian Pac J Cancer Prev 15: 7747-7752, 2014.

6. Süren D, Yıldırım M, Demirpençe Ö, Kaya V, Alikanoğlu AS, Bülbüller N, Yıldız M and Sezer C: The role of high mobility group box 1 (HMGB1) in colorectal cancer. Med Sci Monit 20 530-537, 2014.

7. Ueda M, Takahashi Y, Shinden Y, Sakimura S, Hirata H, Uchi R, Takano Y, Kurashige J, Iguchi T, Eguchi H, et al: Prognostic significance of high mobility group box 1 (HMGB1) expression in patients with colorectal cancer. Anticancer Res 34: 5357-5362, 2014

8. Sims GP, Rowe DC, Rietdijk ST, Herbst R and Coyle AJ: HMGB1 and RAGE in inflammation and cancer. Annu Rev Immunol 28 367-388, 2010

9. Tang D, Kang R, Zeh HJ III and Lotze MT: High-mobility group box 1 and cancer. Biochim Biophys Acta 1799: 131-140, 2010.

10. Yu Y, Xie M, He YL, Xu WQ, Zhu S and Cao LZ: Role of high mobility group box 1 in adriamycin-induced apoptosis in leukemia K562 cells. Ai Zheng 27: 929-933, 2008 (In Chinese).

11. Wild CA, Brandau S, Lotfi R, Mattheis S, Gu X, Lang S and Bergmann C: HMGB1 is overexpressed in tumor cells and promotes activity of regulatory $\mathrm{T}$ cells in patients with head and neck cancer. Oral Oncol 48: 409-416, 2012.

12. Curtin JF, Liu N, Candolfi M, Xiong W, Assi H, Yagiz K, Edwards MR, Michelsen KS, Kroeger KM, Liu C, et al: HMGB1 mediates endogenous TLR2 activation and brain tumor regression. PLoS Med 6: e10, 2009.
13. Zhang W, Tian J and Hao Q: HMGB1 combining with tumorassociated macrophages enhanced lymphangiogenesis in human epithelial ovarian cancer. Tumour Biol 35: 2175-2186, 2014.

14. Zhao M, Yang M, Yang L, et al: HMGB1 regulates autophagy through increasing transcriptional activities of JNK and ERK in human myeloid leukemia cells. BMB Rep 44: 601-606, 2011.

15. Nakamura K, Yoshikawa N, Yamaguchi Y, Kagota S, Shinozuka K and Kunitomo M: Antitumor effect of cordycepin (3'-deoxyadenosine) on mouse melanoma and lung carcinoma cells involves adenosine A3 receptor stimulation. Anticancer Res 26: 43-47, 2006.

16. Zhou X, Meyer CU, Schmidtke P and Zepp F: Effect of cordycepin on interleukin-10 production of human peripheral blood mononuclear cells. Eur J Pharmacol 453: 309-317, 2002.

17. Koç Y, Urbano AG, Sweeney EB and McCaffrey R: Induction of apoptosis by cordycepin in ADA-inhibited TdT-positive leukemia cells. Leukemia 10: 1019-1024, 1996.

18. Chen YH, Wang JY, Pan BS, Mu YF, Lai MS, So EC, Wong TS and Huang BM: Cordycepin enhances cisplatin apoptotic effect through caspase/MAPK pathways in human head and neck tumor cells. Onco Targets Ther 6: 983-998, 2013.

19. Jeong MH, Lee CM, Lee SW, Seo SY, Seo MJ, Kang BW, Jeong YK, Choi YJ, Yang KM and Jo WS: Cordycepinenriched Cordyceps militaris induces immunomodulation and tumor growth delay in mouse-derived breast cancer. Oncol Rep 30: 1996-2002, 2013.

20. Zhang P, Huang C, Fu C, Tian Y, Hu Y, Wang B, Strasner A, Song Y and Song E: Cordycepin (3'-deoxyadenosine) suppressed HMGA2, Twist1 and ZEB1-dependent melanoma invasion and metastasis by targeting miR-33b. Oncotarget 6: 9834-9853, 2015.

21. Yao Z and Shulan Z: Inhibition effect of Guizhi-Fuling-decoction on the invasion of human cervical cancer. J Ethnopharmacol 120: 25-35, 2008.

22. Simon HU, Haj-Yehia A and Levi-Schaffer F: Role of reactive oxygen species (ROS) in apoptosis induction. Apoptosis 5: 415-418, 2000.

23. Herold K, Moser B, Chen Y, et al: Receptor for advanced glycation end products (RAGE) in a dash to the rescue: Inflammatory signals gone awry in the primal response to stress. J Leukoc Biol 82: 204-212, 2007.

24. Grösch S, Tegeder I, Niederberger E, Bräutigam L and Geisslinger G: COX-2 independent induction of cell cycle arrest and apoptosis in colon cancer cells by the selective COX-2 inhibitor celecoxib. FASEB J 15: 2742-2744, 2001.

25. Hirji I, Gupta S, Goren A, et al: Chronic myeloid leukemia (CML): Association of treatment satisfaction, negative medication experience and treatment restrictions with health outcomes, from the patient's perspective. Health Qual Life Outcomes 11: 167, 2013.

26. Jeong JW, Jin CY, Park C, et al: Induction of apoptosis by cordycepin via reactive oxygen species generation in human leukemia cells. Toxicol In Vitro 25: 817-824, 2011.

27. Yuan XL, Chen L, Li MX, et al: Elevated expression of Foxp3 in tumor-infiltrating Treg cells suppresses T-cell proliferation and contributes to gastric cancer progression in a COX-2-dependent manner. Clin Immunol 134: 277-288, 2010.

28. Möbius C, Stein HJ, Spiess C, Becker I, Feith M, Theisen J, Gais P, Jütting U and Siewert JR: COX2 expression, angiogenesis, proliferation and survival in Barrett's cancer. Eur J Surg Oncol 31: 755-759, 2005.

29. Sánchez-Fidalgo S, Martín-Lacave I, Illanes M and Motilva V: Angiogenesis, cell proliferation and apoptosis in gastric ulcer healing. Effect of a selective cox-2 inhibitor. Eur J Pharmacol 505: 187-194, 2004.

30. Okuma Y, Liu K, Wake H, et al: Anti-high mobility group box-1 antibody therapy for traumatic brain injury. Ann Neurol 72: 373-384, 2012.

31. Sessa L, Gatti E, Zeni F, et al: The receptor for advanced glycation end-products (RAGE) is only present in mammals and belongs to a family of cell adhesion molecules (CAMs). PLoS One 9: e86903, 2014.

32. Ola MS, Nawaz M and Ahsan $\mathrm{H}$ : Role of Bcl-2 family proteins and caspases in the regulation of apoptosis. Mol Cell Biochem 351: 41-58, 2011

33. Hoshyar R, Bathaie SZ and Sadeghizadeh M: Crocin triggers the apoptosis through increasing the $\mathrm{Bax} / \mathrm{Bcl}-2$ ratio and caspase activation in human gastric adenocarcinoma, AGS, cells. DNA Cell Biol 32: 50-57, 2013

34. Yang L, Wang P, Wang H, et al: Fucoidan derived from Undaria pinnatifida induces apoptosis in human hepatocellular carcinoma SMMC-7721 cells via the ROS-mediated mitochondrial pathway. Mar Drugs 11: 1961-1976, 2013. 